\title{
The Relationship between Plasma Oxytocin Levels and Social Anxiety Symptoms
}

\author{
Kang-Seob $\mathrm{Oh}^{1 *}$, Eun-Jin $\mathrm{Kim}^{2 *}$, Ju-Won $\mathrm{Ha}^{3}$, Hee-Yeon Woo ${ }^{4}$, \\ Min-Jung Kwon ${ }^{4}$, Dong-Won Shin ${ }^{1}$, Young-Chul Shin ${ }^{1,2}$, and Se-Won Lim ${ }^{1,2}$ \\ 'Department of Psychiatry, Kangbuk Samsung Hospital, Sungkyunkwan University School of Medicine, Seoul, Republic of Korea \\ ${ }^{2}$ Workplace Mental Health Institute, Kangbuk Samsung Hospital, Seoul, Republic of Korea \\ ${ }^{3}$ Yonsei Forest Mental Health Clinic, Seoul, Republic of Korea \\ ${ }^{4}$ Department of Laboratory Medicine, Kangbuk Samsung Hospital, Sungkyunkwan University School of Medicine, Seoul, Republic of Korea
}

Objective The pathophysiology of social anxiety disorder (SAD) is not yet well understood, but previous research has suggested that oxytocin is associated with social behavior and may play a role in human anxiety states and anxiety-related traits. The aim of this study was to investigate the possible relationship between social anxiety symptoms and plasma oxytocin levels.

Methods Twenty-three male patients with SAD and 28 healthy male controls participated in this study. All participants were assessed using the Mini International Neuropsychiatric Interview (MINI) and the Liebowitz Social Anxiety Scale (LSAS). Multivariate regression analysis was performed to identify associations between plasma oxytocin levels and SAD.

Results In multiple regression models, after controlling for age and years of education, we found that higher oxytocin levels were significantly associated with higher total LSAS scores $\left(\mathrm{R}^{2}=0.157\right.$, coefficient $\left.=0.145,95 \% \mathrm{CI}=-0.0005-0.291, \mathrm{p}=0.051\right)$ and fear subscale scores $\left(\mathrm{R}^{2}=0.134\right.$, coefficient $\left.=0.083,95 \% \mathrm{CI}=0.007-0.159, \mathrm{p}=0.034\right)$ in the $\mathrm{SAD}$ group.

Conclusion In this study, increased plasma oxytocin levels were associated with higher social anxiety symptoms among SAD patients, but not among controls. This might be because among SAD patients, higher oxytocin (OT) secretion is an insufficient compensatory attempt to reduce social anxiety symptoms.

Psychiatry Investig 2018;15(11):1079-1086

Key Words Social anxiety disorder, Oxytocin, Liebowitz Social Anxiety Scale, Fear, Avoidance.

\section{INTRODUCTION}

Social anxiety disorder (SAD) is a condition characterized by excessive fear of social interaction, pervasive behavioral avoidance ${ }^{1}$ of social and performance situations, and physiological arousal. ${ }^{2}$ Like many other anxiety disorders, SAD is likely caused by a combination of environmental and genetic factors, including inherited traits, neurotransmitter imbalance, altered brain activity, and negative life experience. ${ }^{1}$ The most consistent findings regarding SAD's neurobiological

Received: June 23, 2017 Revised: June 8, 2018 Accepted: August 31, 2018

$\bowtie$ Correspondence: Se-Won Lim, MD, PhD

Department of Psychiatry, Kangbuk Samsung Hospital, Sungkyunkwan University School of Medicine, 29 Saemunan-ro, Jongno-gu, Seoul 03181, Republic of Korea

Tel: +82-2-2001-2591, Fax: +82-2-2001-2211, E-mail: healthysewon@daum.net

*These authors contributed equally to this work.

(c) This is an Open Access article distributed under the terms of the Creative Commons Attribution Non-Commercial License (https://creativecommons.org/licenses/by$\mathrm{nc} / 4.0$ ) which permits unrestricted non-commercial use, distribution, and reproduction in any medium, provided the original work is properly cited. factors include various neurotransmitters (serotonin, norepinephrine, glutamate, and $\gamma$-aminobutyric acid) and neuropeptides, such as oxytocin (OT), but there are no comprehensive hypotheses yet. ${ }^{3}$ Recently, OT is becoming increasingly considered a prosocial neuropeptide and is a major focus of current social anxiety research. ${ }^{3,4}$

OT is a nine-amino-acid neuropeptide synthesized in the magnocellular and parvocellular neurons of the paraventricular (PVN) and supraoptic (SON) nuclei of the hypothalamus. ${ }^{5} \mathrm{OT}$ is an abundant neuropeptide that is released into the bloodstream from the posterior pituitary, and has a wide spectrum of central and peripheral effects. ${ }^{6}$ OT was first recognized for its involvement in labor and lactation across species and was subsequently found to improve maternal care of offspring, pair bonding formation in various kinds of mammals, sexual behavior, and social behaviors. ${ }^{7-9}$

Previous research that addressed the association between plasma OT levels and anxiety produced inconclusive results. Numerous behavioral studies in animals and humans have 
demonstrated evidence for a role of OT in social affiliative behavior and have suggested that OT can decrease anxiety-like behavior, which may result in an increased ability to respond pro-socially to social interactions. It is likely that the anxiolytic effect of OT is produced through certain mechanisms. First, OT receptors are rich in the amygdala. ${ }^{10,11}$ SAD patients exhibited exaggerated amygdala reactivity to threat-related cues during functional neuroimaging studies ${ }^{12,13}$ and attenuation of this hyperactive amygdala response was observed after intranasal OT administration. ${ }^{14-16}$ Second, OT suppresses the stress hormones of the hypothalamic-pituitary-adrenal (HPA) axis. Intracerebral OT interacts with HPA axis responsiveness, including hypophysiotropic control of adrenocorticotropic hormone (ACTH) release in the stress response. ${ }^{17,18}$ When a stress reaction is provoked either by innate or conditioned fear stimuli, HPA activation increases the production of plasma corticosterone in rodents and cortisol in primates. ${ }^{19} \mathrm{By}$ regulating the HPA axis and amygdala activity, OT plays an important role in modulating fear and anxiety responses through its projections to the brain stem and hypothalamic structures. ${ }^{20}$

Initial human research found similar stress-attenuating effects of OT. Among healthy men, intranasal administration of OT resulted in lower cortisol concentrations during stress exposure compared with a placebo group. ${ }^{21}$ Recently, studies demonstrated that intranasal administration of OT stimulates a wide range of social behaviors, including trust, empathy, and interpersonal interactions, ${ }^{22}$ and promoted the processing of social recognition among healthy males. ${ }^{23,24}$ Although different methodologies are used, Scantamburlo et al. ${ }^{25}$ found a significant negative correlation between plasma OT levels and symptoms of depression and anxiety in a group of 25 depressed patients. Furthermore, previous research by Hoge et al. ${ }^{26}$ reported that after a pro-social task, plasma OT levels were significantly lower among patients with generalized SAD than among healthy controls.

However, in contrast to previous studies of OT's anxiolytic properties, a study of 24 patients with generalized SAD reported that higher social anxiety symptom severity, controlled for age and sex, was related to higher OT levels. ${ }^{27}$ Another study reported that increased OT levels were associated with stronger anxiety symptoms among 29 patients with obsessive compulsive disorder. ${ }^{28}$

Under the hypothesis that OT plays an important role in the modulation of social anxiety, fear response, and avoidance behavior in SAD patients, we investigated the association between plasma OT levels and social anxiety symptoms among SAD male patients and compared them with healthy controls. Since estrogen and progesterone have an important effect on OT gene expression and OT receptors in females, this study included only male subjects.

\section{METHODS}

\section{Participants}

Twenty-three male subjects with SAD and 28 healthy male controls participated in this study. The participants were between 18 and 65 years of age and were recruited from an outpatient clinic at Kangbuk Samsung Hospital. Healthy controls were recruited through hospital advertisements. The Diagnostic and Statistical Manual for Mental Disorders IV $(\mathrm{DSM}-\mathrm{IV})^{29}$ criteria were used for the diagnosis of SAD. The Mini International Neuropsychiatric Interview (MINI) ${ }^{30}$ and the Leibowitz Social Anxiety Scale (LSAS) ${ }^{31}$ were used to assess all patients. The MINI is a validated clinician-administered structured diagnostic interview used to assess major categories of psychiatric disorders. ${ }^{30}$ Exclusion criteria included major depressive disorder, other anxiety disorders, psychotic disorders, substance abuse or dependence, and persistent suicidal ideation within the past six months. Other exclusion criteria included unstable medical illness and the use of dopaminergic medication (both antagonists and agonists). All participants provided written informed consent prior to the procedure, and the study and consent protocol were approved by the ethics committee of Kangbuk Samsung Hospital, Sungkyunkwan University.

\section{Plasma OT analysis}

Venous blood samples $(2 \mathrm{~mL})$ were drawn in EDTA tubes between 10 a.m. and 4 p.m. Participants were asked not to consume any caffeine on the day of blood sampling; alcohol was not permitted in the 24 hours prior to the sampling. Plasma OT levels were measured by competitive immunoassay at baseline. The same amount of $1 \%$ trifluoroacetic acid in purified water was added, and the mixture was centrifuged at $6,000-17,000 \times \mathrm{g}$ for 20 minutes at $4^{\circ} \mathrm{C}$. Extraction Kit S-5000 (Peninsula Laboratories Inc., San Carlos, CA, USA) was used for plasma OT extraction. Plasma samples were stored at $-70^{\circ} \mathrm{C}$ until analysis, which occurred within three months of collection. To prevent laboratory error, each plasma OT sample was analyzed twice, and the mean concentration was used in the statistical analyses. Inter- and intra-assay variability was less than $5 \%$. Outliers greater than $2 \mathrm{SD}$ above the mean were excluded in this analysis.

\section{Anxiety assessment}

Social anxiety severity was measured using the LSAS, a commonly used scale to assess symptoms of social anxiety. ${ }^{32}$ The LSAS is a 24-item clinician-rated scale that measures fear and avoidance in social and performance situations. ${ }^{31}$ The LSAS provides scores on two subscales: fear/anxiety ( 0 , none; 1 , mild; 2 , moderate; and 3, severe) and avoidance behavior (0, 
Table 1. Demographic characteristics of the study population

\begin{tabular}{lcccc}
\hline & Control $(\mathrm{N}=28)$ & $\mathrm{SAD}(\mathrm{N}=23)$ & t or $\chi^{2}$ & $\mathrm{p}$ \\
\hline Age (years) & $25.04 \pm 2.56$ & $40.26 \pm 12.44$ & 5.770 & $<0.001^{*}$ \\
Education (years) & $17.59 \pm 0.75$ & $14.7 \pm 2.12$ & -6.232 & $<0.001^{*}$ \\
Marital status, partnered (\%) & $16(61.5)$ & $10(38.5)$ & 1.239 & $0.266^{\dagger}$ \\
\hline
\end{tabular}

Data are expressed as mean \pm standard deviation or $\mathrm{N}(\%)$. *statistical significance based on Student's t-test, ${ }^{\dagger}$ statistical significance based on Chi-square test, as appropriate. SAD: Social Anxiety Disorder

Table 2. Clinical characteristics of the study population

\begin{tabular}{lcccrrr}
\hline & Control $(\mathrm{N}=28)$ & $\mathrm{SAD}(\mathrm{N}=23)$ & $\mathrm{t}$ & $\mathrm{p}^{\dagger}$ & \multicolumn{1}{c}{$\mathrm{F}$} & \multicolumn{1}{c}{$\mathrm{p}^{\ddagger}$} \\
\hline LSAS, total & $24.75 \pm 16.02$ & $72.83 \pm 29.57$ & 6.999 & $<0.001$ & 14.476 & $<0.001$ \\
LSAS, fear & $14.71 \pm 10.37$ & $38.04 \pm 15.18$ & 6.266 & $<0.001$ & 8.387 & $<0.001$ \\
LSAS, avoidance & $10.04 \pm 7.61$ & $34.78 \pm 15.90$ & 6.848 & $<0.001$ & 19.612 & $<0.001$ \\
Oxytocin $(\mathrm{pg} / \mathrm{mL})^{*}$ & $116.29 \pm 81.1$ & $215.6 \pm 120$ & 3.437 & $<0.001$ & 0.603 & 0.034 \\
\hline
\end{tabular}

Data are expressed as mean \pm standard deviation or $\mathrm{N}(\%) .{ }^{*}$ logarithmic transformed values are used, ${ }^{\dagger}$ statistical significance based on Student's t-test, ${ }^{\ddagger}$ adjusted for age and years of education. LSAS: Liebowitz Social Anxiety Scale

never; 1, occasionally; 2, often; and 3, usually). We used the Korean self-report version of the LSAS, which has been validated and demonstrated to be reliable. ${ }^{33}$

\section{Statistical methods}

Demographic and clinical variables were compared using Student's t-tests for continuous variables and Chi-square tests for categorical variables. The first step of the analysis was to compare mean oxytocin levels by diagnostic group using twotailed t-tests. Differences in plasma OT levels between SAD patients and healthy controls were compared using a MannWhitney U test. To compare plasma OT levels and LSAS scores of SAD and control groups, a univariate analysis of covariance (ANCOVA) was performed, controlling for age and years of education. This was followed by multivariate linear regression modeling to adjust for age and years of education. Within each group, we examined the associations between oxytocin levels and LSAS total scores, fear subscale scores, and avoidance subscale scores. According to the Shapiro-Wilk test, OT data were skewed, so they were log transformed. Statistical analyses were performed using SPSS statistical software, version 18.0 (SPSS, Chicago, IL, USA). The cut-off for statistical significance was set at $\mathrm{p}<0.05$.

\section{RESULTS}

\section{Demographic characteristics of study subjects}

Descriptive demographic characteristics of the study pop-

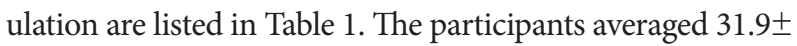
11.41 years of age (range, 20 to 65 years), with significant differences (25.04 \pm 2.56 , control; 40.26 $\pm 12.44, \mathrm{SAD} ; \mathrm{t}=5.770$, $\mathrm{p}<0.001$ ) between the two groups. Although there were no statistically significant differences between the two groups in marital status (61.5\% partnered, control; 38.5\% partnered,

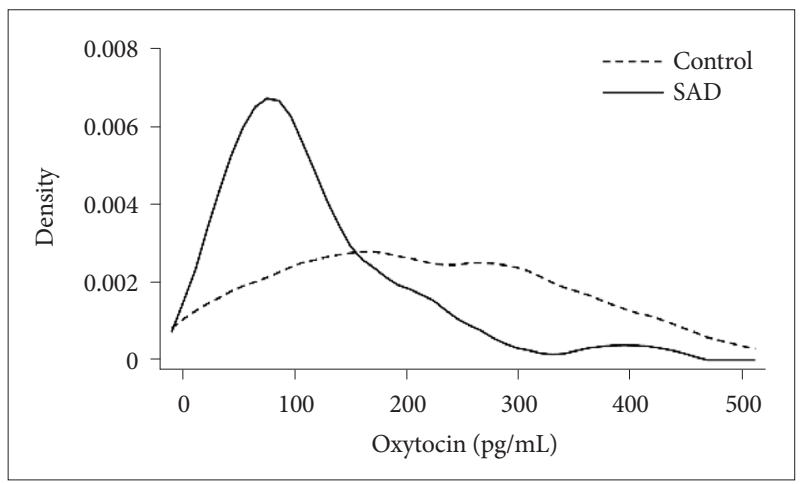

Figure 1. Kernel density plot of plasma Oxytocin levels $(p=0.002)$. SAD: Social anxiety disorder.

$\left.\mathrm{SAD} ; \chi^{2}=1.239, \mathrm{p}=0.266\right)$, the years of education was different between two groups (17.59 \pm 0.75 , control; $14.7 \pm 2.12, \mathrm{SAD}$; $\mathrm{t}=-6.232, \mathrm{p}<0.001)$.

\section{Clinical characteristics of study subjects}

Clinical characteristics of the study population are listed in Table 2. Mean plasma OT levels differed significantly between healthy controls and SAD patients in univariate analyses (116.29 $\pm 81.1 \mathrm{pg} / \mathrm{mL}$, control; $215.6 \pm 120 \mathrm{pg} / \mathrm{mL}, \mathrm{SAD} ; \mathrm{t}=3.437$, $\mathrm{p}<0.001)$. These significant differences between plasma OT levels of SAD patients and controls remained after controlling for age and years of education $(\mathrm{p}=0.034)$. Distribution of plasma OT levels was significantly different between controls and SAD patients [206.38 (104.0-315.0), control; 85.21 (59.98157.84)], SAD; $\mathrm{p}=0.002$ by Mann-Whitney $\mathrm{U}$ test). Figure 1 showed different Kernel density plot of plasma OT levels between two groups.

The mean total and subscale scores of the LSAS for each group were as follows: total score, $24.75 \pm 16.02$, control vs. 72.83 $\pm 29.57, \mathrm{SAD}(\mathrm{t}=6.999, \mathrm{p}<0.001)$; fear subscale score, $14.71 \pm 10.37$, control vs. $38.04 \pm 15.18$, $\mathrm{SAD}(\mathrm{t}=6.266, \mathrm{p}<0.001)$; 
and avoidance subscale score, $10.04 \pm 7.61$, control vs. 34.78 \pm 15.90, $\mathrm{SAD}(\mathrm{t}=6.848, \mathrm{p}<0.001)$.
Relationship between plasma oxytocin levels and social anxiety symptoms

Levels of plasma OT were correlated with LSAS total scores
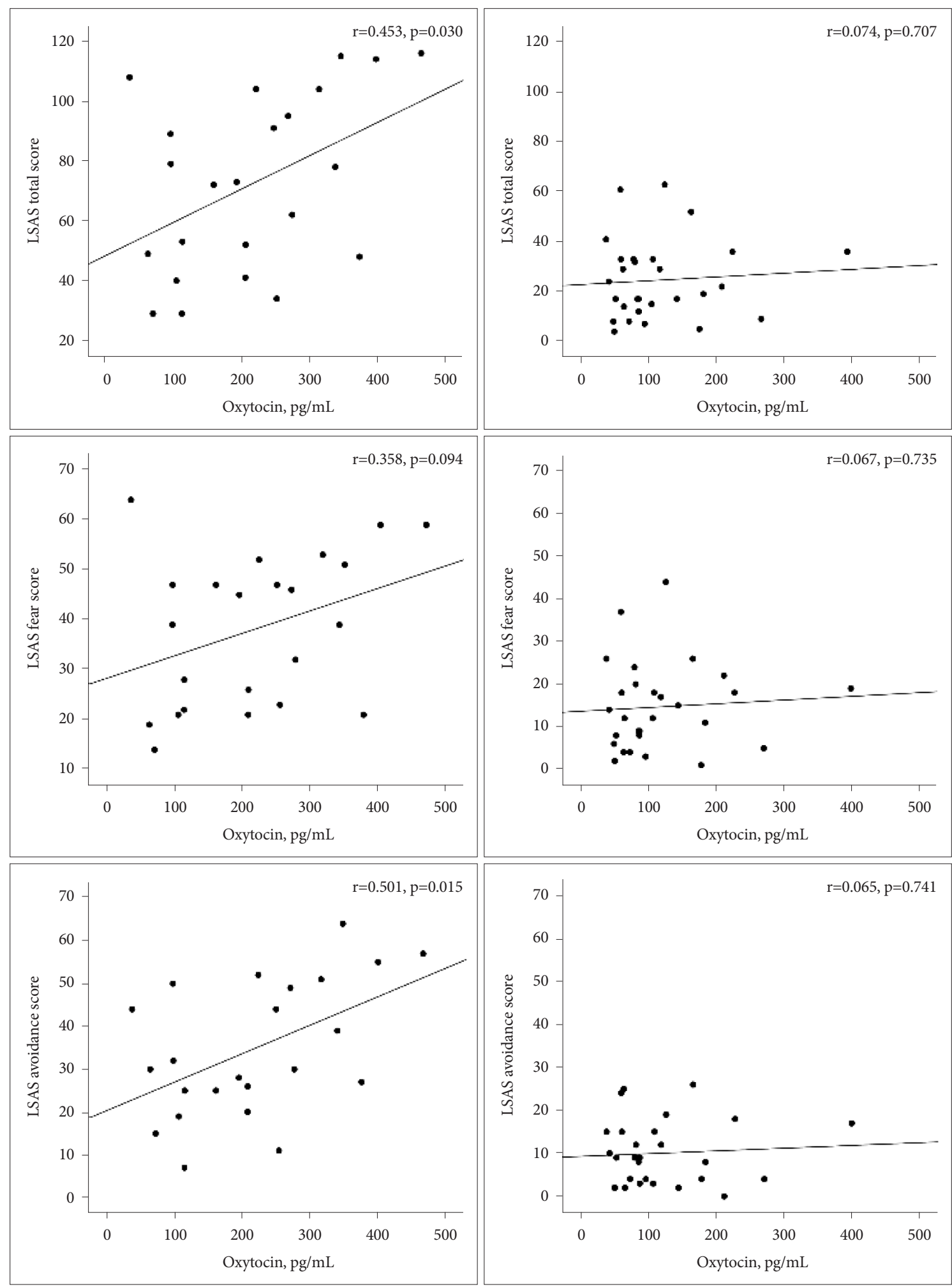

Figure 2. Correlations between plasma OT levels and LSAS total scores, fear scores, and avoidance scores among SAD (left) participants and controls (right). OT: oxytocin, LSAS: Liebowitz Social Anxiety Scale, SAD: Social anxiety disorder. 
$(\mathrm{r}=0.453, \mathrm{p}=0.030)$, fear subscale scores $(\mathrm{r}=0.358, \mathrm{p}=0.094)$, and avoidance subscale scores $(\mathrm{r}=0.501, \mathrm{p}=0.015)$ in the SAD group. Figure 2 showed correlations between plasma OT levels and total scores, avoidance scores, and fear scores on the LSAS among SAD and controls. In multiple linear regression models (Table 3), after controlling for age and years of education, we found that higher OT levels were significantly associated with higher LSAS total scores $\left(\mathrm{R}^{2}=0.157\right.$, coefficient $=$ $0.145,95 \% \mathrm{CI}=-0.0005-0.291, \mathrm{p}=0.051)$ and higher fear subscale scores $\left(\mathrm{R}^{2}=0.134\right.$, coefficient $=0.083,95 \% \mathrm{CI}=0.007-$ $0.159, \mathrm{p}=0.034)$, only in the SAD group. There was no significant association between plasma OT levels and LSAS avoidance subscale scores $\left(\mathrm{R}^{2}=0.186\right.$, coefficient $=0.063,95 \% \mathrm{CI}=-0.014-$ $0.140, p=0.105)$ in the $S A D$ group. In the control group, plasma OT levels were not associated with LSAS total scores $\left(\mathrm{R}^{2}=0.005\right.$, coefficient $\left.=0.014,95 \% \mathrm{CI}=-0.072-0.1, \mathrm{p}=0.736\right)$, fear subscale scores $\left(R^{2}=0.005\right.$, coefficient $=0.008,95 \% C I=$ $-0.047-0.063, p=0.764)$, or avoidance subscale scores $\left(R^{2}=0.004\right.$, coefficient $=0.006,95 \% \mathrm{CI}=-0.035-0.047, \mathrm{p}=0.763$ ), after controlling for age and years of education.

\section{DISCUSSION}

The present study examined the relationship between plasma OT levels and social anxiety symptoms among male SAD patients compared with a healthy male control group. Our results indicate that higher plasma OT levels were associated with higher symptom severity, as measured by the LSAS, only in the SAD patient group. This relationship was observed for both LSAS total scores and fear subscale scores, although not for the avoidance subscale, after controlling for age and years of education. Our finding is inconsistent with previous work,

Table 3. Multivariate linear regression analysis of oxytocin levels

\begin{tabular}{|c|c|c|c|c|c|c|c|c|c|}
\hline & \multicolumn{3}{|c|}{ Unadjusted } & \multicolumn{3}{|l|}{ Model 1} & \multicolumn{3}{|l|}{ Model 2} \\
\hline & B (95\% CI) & $\mathrm{p}$ & $\mathrm{R}^{2}$ & B (95\% CI) & $\mathrm{p}$ & $\mathrm{R}^{2}$ & B (95\% CI) & $\mathrm{p}$ & $\mathrm{R}^{2}$ \\
\hline \multicolumn{10}{|l|}{ SAD } \\
\hline LSAS total & & & 0.205 & & & 0.272 & & & 0.157 \\
\hline Oxytocin & $0.112(0.012-0.211)$ & 0.03 & & $0.146(-0.015-0.486)$ & 0.013 & & $0.145(-0.0005-0.291)$ & 0.051 & \\
\hline Age & & & & $-0.695(-1.770-0.380)$ & 0.193 & & $-0.691(-1.946-0.564)$ & 0.263 & \\
\hline Years of education & & & & & & & $0.044(-7.298-7.385)$ & 0.99 & \\
\hline LSAS fear & & & 0.128 & & & 0.234 & & & 0.134 \\
\hline Oxytocin & $0.045(-0.008-0.099)$ & 0.094 & & $0.067(0.009-0.126)$ & 0.026 & & $0.083(0.007-0.159)$ & 0.034 & \\
\hline Age & & & & $-0.451(-1.017-0.115)$ & 0.112 & & $-0.551(-1.204-0.101)$ & 0.093 & \\
\hline Years of education & & & & & & & $-1.244(-5.063-2.576)$ & 0.504 & \\
\hline LSAS avoidance & & & 0.251 & & & 0.279 & & & 0.186 \\
\hline Oxytocin & $0.066(0.014-0.118)$ & 0.015 & & $0.078(0.019-0.138)$ & 0.013 & & $0.063(-0.014-0.140)$ & 0.105 & \\
\hline Age & & & & $-0.244(-0.819-0.331)$ & 0.388 & & $-0.140(-0.803-0.523)$ & 0.664 & \\
\hline Years of education & & & & & & & $1.287(-2.590-5.165)$ & 0.496 & \\
\hline \multicolumn{10}{|l|}{ Control } \\
\hline LSAS total & & & 0.006 & & & 0.007 & & & 0.005 \\
\hline Oxytocin & $0.015(-0.065-0.094)$ & 0.707 & & $0.016(-0.066-0.098)$ & 0.697 & & $0.014(-0.072-0.100)$ & 0.736 & \\
\hline Age & & & & $-0.220(-2.814-2.374)$ & 0.863 & & $-0.185(-3.844-3.475)$ & 0.918 & \\
\hline Years of education & & & & & & & $0.061(-12.586-12.708)$ & 0.992 & \\
\hline LSAS fear & & & 0.004 & & & 0.007 & & & 0.005 \\
\hline Oxytocin & $0.009(-0.043-0.060)$ & 0.735 & & $0.009(-0.044-0.062)$ & 0.718 & & $0.008(-0.047-0.063)$ & 0.764 & \\
\hline Age & & & & $-0.192(-1.872-1.487)$ & 0.815 & & $-0.216(-2.577-2.145)$ & 0.851 & \\
\hline Years of education & & & & & & & $0.338(-7.820-8.497)$ & 0.932 & \\
\hline LSAS avoidance & & & 0.004 & & & 0.004 & & & 0.004 \\
\hline Oxytocin & $0.006(-0.032-0.044)$ & 0.741 & & $0.006(-0.033-0.045)$ & 0.744 & & $0.006(-0.035-0.047)$ & 0.763 & \\
\hline Age & & & & $-0.028(-1.261-1.206)$ & 0.963 & & $0.031(-1.715-1.778)$ & 0.971 & \\
\hline Years of education & & & & & & & $-0.277(-6.311-5.757)$ & 0.925 & \\
\hline
\end{tabular}

B (95\% CI): coefficient and 95\% confidence interval, Model 1: adjusted for age, Model 2: adjusted for age and years of education. LSAS: Liebowitz Social Anxiety Scale 
which found that OT plays a key role in the reduction of anxiety and enhances the buffering effect of social support during stressful procedures. ${ }^{21}$ Our results evoke interest regarding other aspects of OT in social anxiety.

As mentioned above, inspection of the previous literature reveals that the effects of OT on social anxiety are mixed; some studies suggest that OT is anxiolytic, and others emphasize OT's anxiogenic effects. Humans exposed to OT experience enhanced in-group trust and are better able to recognize emotions from others' facial expressions. ${ }^{34,35} \mathrm{OT}$ and its receptors have buffering effects on neuroendocrine and autonomic activities in response to threat or danger and might operate as an adaptive response to psychosocial stressors. In contrast to OT's anxiolytic role in social interactions, MacDonald et al..$^{36}$ observed that OT causes an increase in anxiety during 20min psychotherapy sessions among outpatients with major depressive disorder. Marazziti et al. ${ }^{37}$ reported that attachment-related anxiety and OT plasma levels are positively and significantly linked in humans, and the results of a self-report questionnaire measuring adult romantic attachment suggested that higher OT levels produced higher scores on an anxiety scale with regard to experiences in close relationships. In a study involving autistic children, higher OT levels were significantly associated with social and developmental impairment. ${ }^{38}$ In line with the above findings, higher OT levels were closely related to dissatisfaction with social relationships and higher social anxiety symptom severity among the SAD patients compared with healthy controls. ${ }^{27}$

Whether different experimental factors linked to OT might play a causal role in the unexpected anxiogenic effects in social anxiety disorders remains to be determined. A simple OT deficit or excess model is inadequate to explain this mechanism. Further adjustment for other regulating factors that may influence these results, including baseline characteristics, should be performed. For example, high plasma OT levels in SAD may reflect underlying disruptions in receptors or in an extended form of $\mathrm{OT}^{39}$ considered a precursor for OT, resulting in secondary OT dysregulation. Also, emotional and physical stressors, such as exposure to an invasive blood sampling procedure, trigger the increased release of OT from both the SON and the PVN among SAD patients relative to controls. ${ }^{40,41}$

From a neurobiological point of view, repeated-exposure therapy to reduce SAD patients' fear and anxiety may have a close association with high plasma OT levels in SAD. When anxiety and fear occur in response to a signal threat or social situation, SAD patients exhibit excessive reactivity in the amygdala and the insula. ${ }^{42,43}$ Activation of the fear system leads to a series of behavioral and physiological symptoms that enable the person to escape from and avoid danger, so-called escape and avoidance behaviors, respectively. ${ }^{1}$ Avoidance of stressful situations has been found to maintain SAD symptoms, whereas a reduction in avoidance behavior has been associated with reduced anxiety. ${ }^{44}$ To reduce social anxiety, SAD patients are treated with exposure therapy, during which they are exposed to social situations until the fear eventually subsides. ${ }^{45}$ During repeated-exposure therapy, changes in neural processing occur, including increases in prefrontal activity in conjunction with decreases in activity of the amygdala. ${ }^{46,47}$ It is also reported that the local oxytocinergic fibers located in the central nucleus of the amygdala release increased OT during stressful or anxiety-provoking situations. ${ }^{48}$ Therefore, we can presume that the high plasma OT levels among the SAD group may be a compensatory physiological mechanism attempting to reduce anxiety and facilitate social behavior.

This study has several limitations. First, the small sample size limited our ability to evaluate and control potential confounding factors. In addition, a small sample size may leads to reduced likelihood of detecting real associations between plasma OT levels and social anxiety symptoms. Second, this study included men only, limiting the generalizability of our results. Several studies suggest that sex plays a specific role in the association between plasma OT and anxiety. Future research is needed to investigate whether gender moderates the relationship between OT and anxiety symptoms. Third, the OT concentrations were measured only in peripheral plasma. It is still unclear whether plasma levels accurately reflect concentrations in the brain, since it appears that central and peripheral OT derive from different populations of hypothalamic cell. ${ }^{49,50}$ Fourth, there are technical and methodological limitations to OT analysis. The circadian rhythm of OT was reported in a prior study. ${ }^{51}$ OT peaks in the morning and declines in the early afternoon. In this study, plasma OT level was collected over a wide range of times (from 9am to $4 \mathrm{pm}$ ). Although we did not strictly control the sampling time of OT, the time windows of OT sampling for both patients and the control group were identical. Fifth, there are significant age differences between the two groups. Only a few studies have investigated effects of age on the oxytocin system. In one human postmortem study, evidence of reduced numbers of oxytonergic cells in the paraventricular nuclei of elderly was reported, ${ }^{52}$ but others found no changes. ${ }^{53}$ Human studies about the relationship between age and oxytocin level have so far provided inconclusive result. However, we cannot exclude the possibility that the age difference might have influenced the result of this study. Although we have statistically controlled the age differences, the significant difference in age between two groups can be an important limitation of this study. Sixth, this study did not assess important variables known to be associated with OT levels such as general over- 
all health, pre-study blood test, body mass index and pre-existing medical conditions. Therefore, future researches that include these variables are needed to determine the association between plasma OT levels and social anxiety symptoms. Seventh, the cross-sectional nature of our study makes it difficult to implicate a causal relationship from the results. Prospective longitudinal studies might help to model this possible interaction linking social anxiety symptoms and plasma OT concentrations.

Given the paucity of results on the possible roles of OT in $\mathrm{SAD}$, this study explores the potential relationships between the OT plasma levels and SAD symptoms. Data from this study support the idea that OT has a specific effect on individuals with SAD. However, human studies in this field are sparse, and further clinical studies are needed.

\section{REFERENCES}

1. Toth I, Neumann ID. Animal models of social avoidance and social fear. Cell Tissue Res 2013;354:107-118.

2. Aderka IM, McLean CP, Huppert JD, Davidson JR, Foa EB. Fear, avoidance and physiological symptoms during cognitive-behavioral therapy for social anxiety disorder. Behav Res Ther 2013;51:352-358.

3. Marazziti D, Abelli M, Baroni S, Carpita B, Ramacciotti CE, Dell'Osso L. Neurobiological correlates of social anxiety disorder: an update. CNS Spectr 2015;20:100-111.

4. Stevens FL, Weisman O, Feldman R, Hurley RA, Taber KH. Oxytocin and behavior: evidence for effects in the brain. J Neuropsychiatry Clin Neurosci 2013;25:96-102.

5. Sofroniew MV. Morphology of vasopressin and oxytocin neurones and their central and vascular projections. Prog Brain Res 1983;60:101114.

6. Ozsoy S, Esel E, Kula M. Serum oxytocin levels in patients with depression and the effects of gender and antidepressant treatment. Psychiatry Res 2009;169:249-252.

7. Uvnas-Moberg K. Oxytocin may mediate the benefits of positive social interaction and emotions. Psychoneuroendocrinology 1998;23:819-835.

8. Ross HE, Young LJ. Oxytocin and the neural mechanisms regulating social cognition and affiliative behavior. Front Neuroendocrinol 2009; 30:534-547.

9. Pedersen CA, Caldwell JD, Peterson G, Walker CH, Mason GA. Oxytocin activation of maternal behavior in the rat. Ann N Y Acad Sci 1992; 652:58-69.

10. Huber D, Veinante P, Stoop R. Vasopressin and oxytocin excite distinct neuronal populations in the central amygdala. Science 2005;308:245248.

11. Gimpl G, Fahrenholz F. The oxytocin receptor system: structure, function, and regulation. Physiol Rev 2001;81:629-683.

12. Evans KC, Wright CI, Wedig MM, Gold AL, Pollack MH, Rauch SL. A functional MRI study of amygdala responses to angry schematic faces in social anxiety disorder. Depress Anxiety 2008;25:496-505.

13. Goldin PR, Manber T, Hakimi S, Canli T, Gross JJ. Neural bases of social anxiety disorder: emotional reactivity and cognitive regulation during social and physical threat. Arch Gen Psychiatry 2009;66:170-180.

14. Labuschagne I, Phan KL, Wood A, Angstadt M, Chua P, Heinrichs M, et al. Medial frontal hyperactivity to sad faces in generalized social anxiety disorder and modulation by oxytocin. Int J Neuropsychopharmacol 2012;15:883-896.

15. Dodhia S, Hosanagar A, Fitzgerald DA, Labuschagne I, Wood AG, Nathan PJ, et al. Modulation of resting-state amygdala-frontal functional connectivity by oxytocin in generalized social anxiety disorder. Neuropsychopharmacology 2014;39:2061-2069.

16. Labuschagne I, Phan KL, Wood A, Angstadt M, Chua P, Heinrichs M, et al. Oxytocin attenuates amygdala reactivity to fear in generalized social anxiety disorder. Neuropsychopharmacology 2010;35:2403-2413.

17. Amico JA, Mantella RC, Vollmer RR, Li X. Anxiety and stress responses in female oxytocin deficient mice. J Neuroendocrinol 2004;16:319-324.

18. Neumann ID. Involvement of the brain oxytocin system in stress coping: interactions with the hypothalamo-pituitary-adrenal axis. Prog Brain Res 2002;139:147-162.

19. Miller MW, McKinney AE, Kanter FS, Korte KJ, Lovallo WR. Hydrocortisone suppression of the fear-potentiated startle response and posttraumatic stress disorder. Psychoneuroendocrinology 2011;36:970-980.

20. Wojciak P, Remlinger-Molenda A, Rybakowski J. The role of oxytocin and vasopressin in central nervous system activity and mental disorders. Psychiatr Pol 2012;46:1043-1052.

21. Heinrichs M, Baumgartner T, Kirschbaum C, Ehlert U. Social support and oxytocin interact to suppress cortisol and subjective responses to psychosocial stress. Biol Psychiatry 2003;54:1389-1398.

22. Kosfeld M, Heinrichs M, Zak PJ, Fischbacher U, Fehr E. Oxytocin increases trust in humans. Nature 2005;435:673-676.

23. Rimmele U, Hediger K, Heinrichs M, Klaver P. Oxytocin makes a face in memory familiar. J Neurosci 2009;29:38-42.

24. Evans SL, Dal Monte O, Noble P, Averbeck BB. Intranasal oxytocin effects on social cognition: a critique. Brain Res 2014;1580:69-77.

25. Scantamburlo G, Hansenne M, Fuchs S, Pitchot W, Marechal P, Pequeux $\mathrm{C}$, et al. Plasma oxytocin levels and anxiety in patients with major depression. Psychoneuroendocrinology 2007;32:407-410.

26. Hoge EA, Lawson EA, Metcalf CA, Keshaviah A, Zak PJ, Pollack MH, et al. Plasma oxytocin immunoreactive products and response to trust in patients with social anxiety disorder. Depress Anxiety 2012;29:924-930.

27. Hoge EA, Pollack MH, Kaufman RE, Zak PJ, Simon NM. Oxytocin levels in social anxiety disorder. CNS Neurosci Ther 2008;14:165-170.

28. Leckman JF, Goodman WK, North WG, Chappell PB, Price LH, Pauls DL, et al. Elevated cerebrospinal fluid levels of oxytocin in obsessivecompulsive disorder. Comparison with Tourette's syndrome and healthy controls. Arch Gen Psychiatry 1994;51:782-792.

29. American Psychiatric Association. Diagnostic and Stastical Manual of Mental Disorders. Washington DC: American Psychiatric Association; 1994.

30. Sheehan DV, Lecrubier Y, Sheehan KH, Amorim P, Janavs J, Weiller E, et al. The Mini-International Neuropsychiatric Interview (M.I.N.I.): the development and validation of a structured diagnostic psychiatric interview for DSM-IV and ICD-10. J Clin Psychiatry 1998;59(Suppl 20):2233; quiz 34-57.

31. Heimberg RG, Horner KJ, Juster HR, Safren SA, Brown EJ, Schneier FR, et al. Psychometric properties of the Liebowitz Social Anxiety Scale. Psychol Med 1999;29:199-212.

32. Liebowitz MR, Gorman JM, Fyer AJ, Klein DF. Social phobia. Review of a neglected anxiety disorder. Arch Gen Psychiatry 1985;42:729-736.

33. Yu ES, Ahn CI, Park KH. Factor structure and diagnostic efficiency of a Korean version of the Liebowitz social anxiety scale. Korean J Clin Psychol 2007;26:251-270.

34. Bartz JA, Zaki J, Bolger N, Ochsner KN. Social effects of oxytocin in humans: context and person matter. Trends Cogn Sci 2011;15:301-309.

35. Van Ijzendoorn MH, Bakermans-Kranenburg MJ. A sniff of trust: metaanalysis of the effects of intranasal oxytocin administration on face recognition, trust to in-group, and trust to out-group. Psychoneuroendocrinology 2012;37:438-443.

36. MacDonald K, MacDonald TM, Brune M, Lamb K, Wilson MP, Golshan S, et al. Oxytocin and psychotherapy: a pilot study of its physiological, behavioral and subjective effects in males with depression. Psychoneuroendocrinology 2013;38:2831-2843.

37. Marazziti D, Dell'Osso B, Baroni S, Mungai F, Catena M, Rucci P, et al. A relationship between oxytocin and anxiety of romantic attachment. 
Clin Pract Epidemiol Ment Health 2006;2:28

38. Modahl C, Green L, Fein D, Morris M, Waterhouse L, Feinstein C, et al. Plasma oxytocin levels in autistic children. Biol Psychiatry 1998;43: 270-277.

39. Green L, Fein D, Modahl C, Feinstein C, Waterhouse L, Morris M. Oxytocin and autistic disorder: alterations in peptide forms. Biol Psychiatry 2001;50:609-613.

40. Wotjak CT, Ganster J, Kohl G, Holsboer F, Landgraf R, Engelmann M. Dissociated central and peripheral release of vasopressin, but not oxytocin, in response to repeated swim stress: new insights into the secretory capacities of peptidergic neurons. Neuroscience 1998;85:1209-1222.

41. Neumann ID. Stimuli and consequences of dendritic release of oxytocin within the brain. Biochem Soc Trans 2007;35:1252-1257.

42. Davis M, Whalen PJ. The amygdala: vigilance and emotion. Mol Psychiatry 2001;6:13-34.

43. Etkin A, Wager TD. Functional neuroimaging of anxiety: a meta-analysis of emotional processing in PTSD, social anxiety disorder, and specific phobia. Am J Psychiatry 2007;164:1476-1488.

44. McManus F, Sacadura C, Clark DM. Why social anxiety persists: an experimental investigation of the role of safety behaviours as a maintaining factor. J Behav Ther Exp Psychiatry 2008;39:147-161.

45. Goossens L, Sunaert S, Peeters R, Griez EJ, Schruers KR. Amygdala hyperfunction in phobic fear normalizes after exposure. Biol Psychiatry 2007;62:1119-1125.

46. Hauner KK, Mineka S, Voss JL, Paller KA. Exposure therapy triggers lasting reorganization of neural fear processing. Proc Natl Acad Sci U S A 2012;109:9203-9208.

47. Vouimba RM, Maroun M. Learning-induced changes in mPFC-BLA connections after fear conditioning, extinction, and reinstatement of fear. Neuropsychopharmacology 2011;36:2276-2285.

48. Gouzenes L, Desarmenien MG, Hussy N, Richard P, Moos FC. Vasopressin regularizes the phasic firing pattern of rat hypothalamic magnocellular vasopressin neurons. J Neurosci 1998;18:1879-1885.

49. Insel TR, Shapiro LE. Oxytocin receptor distribution reflects social organization in monogamous and polygamous voles. Proc Natl Acad Sci U S A 1992;89:5981-5985.

50. Keverne EB, Kendrick KM. Oxytocin facilitation of maternal behavior in sheep. Ann N Y Acad Sci 1992;652:83-101.

51. Amico JA, Levin SC, Cameron JL. Circadian rhythm of oxytocin in the cerebrospinal fluid of rhesus and cynomolgus monkeys: effects of castration and adrenalectomy and presence of a caudal-rostral gradient. Neuroendocrinology 1989;50:624-632.

52. Calza L, Pozza M, Coraddu F, Farci G, Giardino L. Hormonal influences on brain ageing quality: focus on corticotropin releasing hormone-, vasopressin- and oxytocin-immunoreactive neurones in the human brain. J Neural Transm (Vienna) 1997;104:1095-1100.

53. Wierda M, Goudsmit E, Van der Woude PF, Purba JS, Hofman MA, Bogte $\mathrm{H}$, et al. Oxytocin cell number in the human paraventricular nucleus remains constant with aging and in Alzheimer's disease. Neurobiol Aging 1991;12:511-516. 